International Journal of Pure and Applied Mathematics

Volume 94 No. 4 2014, 489-499

ISSN: 1311-8080 (printed version); ISSN: 1314-3395 (on-line version)

url: http://www.ijpam.eu

doi: http://dx.doi.org/10.12732/ijpam.v94i4.4

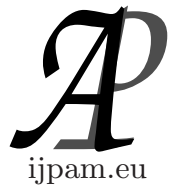

\title{
DELAY-RANGE-DEPENDENT MEAN SQUARE STABILITY OF STOCHASTIC SYSTEMS WITH INTERVAL TIME-VARYING DELAYS
}

\author{
Manlika Rajchakit ${ }^{1}$, Grienggrai Rajchakit ${ }^{2} \S$ \\ ${ }^{1,2}$ Department of Mathematics and Statistics \\ Maejo University \\ Chiangmai, 50290, THAILAND
}

\begin{abstract}
This paper is concerned with mean square exponential stability of stochastic systems with interval time-varying delays. The time delay is any continuous function belonging to a given interval. By constructing a suitable augmented Lyapunov-Krasovskii functional combined with Leibniz-Newton's formula, new delay-dependent sufficient conditions for the mean square exponential stability of the stochastic systems are first established in terms of LMIs.
\end{abstract}

AMS Subject Classification: 15A09, 52A10, 74M05, 93D05

Key Words: mean square exponential stability, stochastic systems, interval delay, Lyapunov function, linear matrix inequalities

\section{Introduction}

The analysis of stochastic systems with respect to mean square stability of their equilibria has attracted many researchers. Such systems occur in a large number of applications as in Physics, Optics or Mechanical Engineering. Often, these systems can generally be written as systems of stochastic differential equations (SDEs). There stability examinations play an essential role in judgement on qualitative behaviour of natural processes.

Received: January 27, 2014

(C) 2014 Academic Publications, Ltd. url: www.acadpubl.eu

${ }^{\S}$ Correspondence author 
The concept of mean square stability is one of the most attractive and feasible ones within the large branch of stability analysis. Due to facilities of modern computers and progress in numerical analysis of stochastic differential equations (SDEs), the interest in mean square stability analysis has come up once again. The basic questions for any numerical algorithm are accuracy and stability. The question of accuracy has been worked out well. However, the question of stability is fairly underdeveloped and still in its very beginning, despite of a number of recent contributions. These contributions exclusively deal with numerical stability analysis with respect to linear test equations in one dimension. The problem of the stability of dynamic systems is one of the basic problems in the control theory. To describe the uncertain parameters and excitations appearing in real dynamic systems in corresponding models usually the stochastic differential equations are used. Developments on the stability of stochastic dynamic systems can be found. An important class of nonlinear control systems, called bilinear control systems, are systems described by stochastic differential equations containing terms of products of state and control variables. The study of bilinear systems began in the late 1960s and has continued from its need in applications (many real world systems appearing in economy, biology, chemistry, biochemistry, physics and engineering can be approximated by bilinear models). Stability analysis of linear systems with time-varying delays $\dot{x}(t)=A x(t)+D x(t-h(t))$ is fundamental to many practical problems and has received considerable attention [1-15]. Most of the known results on this problem are derived assuming only that the time-varying delay $h(t)$ is a continuously differentiable function, satisfying some boundedness condition on its derivative: $\dot{h}(t) \leq \delta<1$. In delay-dependent stability criteria, the main concerns is to enlarge the feasible region of stability criteria in given timedelay interval. Interval time-varying delay means that a time delay varies in an interval in which the lower bound is not restricted to be zero. By constructing a suitable augmented Lyapunov functional and utilizing free weight matrices, some less conservative conditions for asymptotic stability are derived in [16-21] for systems with time delay varying in an interval. However, the shortcoming of the method used in these works is that the delay function is assumed to be differential and its derivative is still bounded: $\dot{h}(t) \leq \delta$. To the best of our knowledge, interval time-varying delay and mean square exponential stability of stochastic systems, non-differentiable time-varying delays have not been fully studied yet (see, e.g., [21-26] and the references therein), which are important in both theories and applications. This motivates our research.

This paper gives the improved results for the mean square exponential stability of stochastic systems with interval time-varying delay. The time delay is 
assumed to be a time-varying continuous function belonging to a given interval, but not necessary to be differentiable. By constructing augmented Lyapunov functional combined with LMI technique, we propose new criteria for the mean square exponential stability of stochastic systems with interval time-varying delay. The delay-dependent mean square exponential stability of stochastic systems with interval time-varying delay conditions are formulated in terms of LMIs.

The outline of the paper is as follows. Section 2 presents definitions and some well-known technical propositions needed for the proof of the main result. LMI delay-dependent mean square exponential stability of stochastic systems with interval time-varying delay criteria showing the effectiveness of the result are presented in Section 3. The paper ends with conclusions and cited references.

\section{Preliminaries}

The following notations will be used in this paper. $R^{+}$denotes the set of all real non-negative numbers; $R^{n}$ denotes the $n$-dimensional space with the scalar product $\langle.,$.$\rangle and the vector norm \|.\| ; M^{n \times r}$ denotes the space of all matrices of $(n \times r)$-dimensions; $A^{T}$ denotes the transpose of matrix $A ; A$ is symmetric if $A=A^{T} ; I$ denotes the identity matrix; $\lambda(A)$ denotes the set of all eigenvalues of $A ; \lambda_{\min / \max }(A)=\min / \max \{\operatorname{Re} \lambda ; \lambda \in \lambda(A)\} ; x_{t}:=\{x(t+s)$ : $s \in[-h, 0]\},\left\|x_{t}\right\|=\sup _{s \in[-h, 0]}\|x(t+s)\| ; C\left([0, t], R^{n}\right)$ denotes the set of all $R^{n}$-valued continuous functions on $[0, t]$; Matrix $A$ is called semi-positive definite $(A \geq 0)$ if $\langle A x, x\rangle \geq 0$, for all $x \in R^{n} ; A$ is positive definite $(A>0)$ if $\langle A x, x\rangle>0$ for all $x \neq 0 ; A>B$ means $A-B>0$. * denotes the symmetric term in a matrix.

Consider a stochastic system with interval time-varying delay of the form

$$
\begin{aligned}
& \dot{x}(t)=A x(t)+D x(t-h(t))+\sigma(x(t), x(k-h(t)), t) \omega(t), \quad t \in R^{+}, \\
& x(t)=\phi(t), t \in\left[-h_{2}, 0\right],
\end{aligned}
$$

where $x(t) \in R^{n}$ is the state; $A, D \in M^{n \times n}$, and $\phi(t) \in C\left(\left[-h_{2}, 0\right], R^{n}\right)$ is the initial function with the norm $\|\phi\|=\sup _{s \in\left[-h_{2}, 0\right]}\|\phi(s)\|$; The time-varying delay function $h(t)$ satisfies

$$
0 \leq h_{1} \leq h(t) \leq h_{2}, \quad t \in R^{+} .
$$

$\omega(k)$ is a scalar Wiener process (Brownian Motion) on $(\Omega, \mathcal{F}, \mathcal{P})$ with

$$
E[\omega(t)]=0, \quad E\left[\omega^{2}(t)\right]=1, \quad E[\omega(i) \omega(j)]=0(i \neq j),
$$


and $\sigma: R^{n} \times R^{n} \times R \rightarrow R^{n}$ is the continuous function, and is assumed to satisfy that

$$
\begin{aligned}
& \sigma^{T}(x(t), x(t-h(t)), t) \sigma(x(t), x(t-h(t)), t) \leq \rho_{1} x^{T}(t) x(t) \\
& +\rho_{2} x^{T}(t-h(t)) x(t-h(t)), \quad x(t), x(t-h(t)) \in R^{n},
\end{aligned}
$$

where $\rho_{1}>0$ and $\rho_{2}>0$ are known constant scalars. For simplicity, we denote $\sigma(x(t), x(t-h(t)), t)$ by $\sigma$, respectively.

The stability problem for stochastic system (1) is to makes the system mean square exponential stability.

Definition 1. Given $\alpha>0$. The stochastic system (1) is $\alpha$-exponentially stable in the mean square if there exists a positive number $N>0$ such that every solution $x(t, \phi)$ of the system satisfies the following condition:

$$
\exists N>0: \quad E\{\|x(t, \phi)\|\} \leq E\left\{N e^{-\alpha t}\|\phi\|\right\}, \quad \forall t \in R^{+} .
$$

We end this section with the following technical well-known propositions, which will be used in the proof of the main results.

Proposition 1. (Cauchy inequality) For any symmetric positive definite matrix $N \in M^{n \times n}$ and $a, b \in R^{n}$ we have

$$
\pm a^{T} b \leq a^{T} N a+b^{T} N^{-1} b .
$$

Proposition 2. [27] For any symmetric positive definite matrix $M \in$ $M^{n \times n}$, scalar $\gamma>0$ and vector function $\omega:[0, \gamma] \rightarrow R^{n}$ such that the integrations concerned are well defined, the following inequality holds

$$
\left(\int_{0}^{\gamma} \omega(s) d s\right)^{T} M\left(\int_{0}^{\gamma} \omega(s) d s\right) \leq \gamma\left(\int_{0}^{\gamma} \omega^{T}(s) M \omega(s) d s\right) .
$$

Proposition 3. [27] Let E, $H$ and $F$ be any constant matrices of appropriate dimensions and $F^{T} F \leq I$. For any $\epsilon>0$, we have

$$
E F H+H^{T} F^{T} E^{T} \leq \epsilon E E^{T}+\epsilon^{-1} H^{T} H .
$$

Proposition 4. (Schur complement lemma [27]). Given constant matrices $X, Y, Z$ with appropriate dimensions satisfying $X=X^{T}, Y=Y^{T}>0$. Then $X+Z^{T} Y^{-1} Z<0$ if and only if

$$
\left(\begin{array}{cc}
X & Z^{T} \\
Z & -Y
\end{array}\right)<0 \quad \text { or } \quad\left(\begin{array}{cc}
-Y & Z \\
Z^{T} & X
\end{array}\right)<0 .
$$




\section{Main Results}

Let us set

$$
\begin{aligned}
\mathcal{M} & =\left(\begin{array}{ccccc}
M_{11} & M_{12} & M_{13} & M_{14} & M_{15} \\
* & M_{22} & 0 & 0 & 0 \\
* & * & M_{33} & 0 & 0 \\
* & * & * & M_{44} & M_{45} \\
* & * & * & * & M_{55}
\end{array}\right), \\
\lambda_{1} & =\lambda_{\min }(P), \lambda_{2}=\lambda_{\max }(P)+2 h_{2}^{2} \lambda_{\max }(R), \\
M_{11} & =A^{T} P+P A+2 \alpha P-e^{-2 \alpha h_{1}} R-e^{-2 \alpha h_{2}} R+2 \rho_{1}, \\
M_{12} & =e^{-2 \alpha h_{1}} R, M_{13}=e^{-2 \alpha h_{2}} R, M_{14}=P D, M_{15}=-S_{2} A, \\
M_{22} & =-e^{-2 \alpha h_{1}} R, M_{33}=-e^{-2 \alpha h_{2}} R, M_{44}=-S_{1} D+2 \rho_{2}, \\
M_{45} & =S_{1}-S_{2} D, M_{55}=S_{2}+S_{2}^{T}+h_{1}^{2} R+h_{2}^{2} R .
\end{aligned}
$$

The main result of this paper is summarized in the following theorem.

Theorem 1. Given $\alpha>0$. The zero solution of the stochastic system (1) is $\alpha$-exponentially stable in the mean square if there exist symmetric positive definite matrices $P, R$, and matrices $S_{i}, i=1,2$ such that satisfying the following conditions

$$
\mathcal{M}<0 .
$$

Moreover, the solution $x(t, \phi)$ of the stochastic system satisfies

$$
E[\|x(t, \phi)\|] \leq E\left[\sqrt{\frac{\lambda_{2}}{\lambda_{1}}} e^{-\alpha t}\|\phi\|\right], \quad \forall t \in R^{+} .
$$

Proof. We consider the following Lyapunov-Krasovskii functional for the system (1)

$$
V\left(t, x_{t}\right)=\sum_{i=1}^{3} V_{i}
$$

where

$$
\begin{aligned}
& V_{1}=x^{T}(t) P x(t) \\
& V_{2}=h_{1} \int_{-h_{1}}^{0} \int_{t+s}^{t} e^{2 \alpha(\tau-t)} \dot{x}^{T}(\tau) R \dot{x}(\tau) d \tau d s \\
& V_{3}=h_{2} \int_{-h_{2}}^{0} \int_{t+s}^{t} e^{2 \alpha(\tau-t)} \dot{x}^{T}(\tau) R \dot{x}(\tau) d \tau d s .
\end{aligned}
$$


It easy to check that

$$
E\left[\lambda_{1}\|x(t)\|^{2}\right] \leq E\left[V\left(t, x_{t}\right)\right] \leq E\left[\lambda_{2}\left\|x_{t}\right\|^{2}\right], \quad \forall t \geq 0
$$

Taking the derivative of $V_{1}$ along the solution of system (1) and taking the mathematical expectation, we obtained

$$
\begin{aligned}
E\left[\dot{V}_{1}\right] & =E\left[2 x^{T}(t) P \dot{x}(t)\right] \\
& =E\left[x^{T}(t)\left[A^{T} P+A P\right] x(t)+2 x^{T}(t) P D x(t-h(t))+2 x^{T}(t) P \sigma \omega(t)\right] ; \\
E\left[\dot{V}_{2}\right] & =E\left[h_{1}^{2} \dot{x}^{T}(t) R \dot{x}(t)-h_{1} e^{-2 \alpha h_{1}} \int_{t-h_{1}}^{t} \dot{x}^{T}(s) R \dot{x}(s) d s-2 \alpha V_{2}\right] ; \\
E\left[\dot{V}_{3}\right] & =E\left[h_{2}^{2} \dot{x}^{T}(t) R \dot{x}(t)-h_{2} e^{-2 \alpha h_{2}} \int_{t-h_{2}}^{t} \dot{x}^{T}(s) R \dot{x}(s) d s-2 \alpha V_{3}\right] .
\end{aligned}
$$

Applying Proposition 2 and the Leibniz - Newton formula, we have

$$
\begin{aligned}
-h_{i} \int_{t-h_{i}}^{t} \dot{x}^{T}(s) R \dot{x}(s) d s \leq & {\left[\int_{t-h_{i}}^{t} \dot{x}(s) d s\right]^{T} R\left[\int_{t-h_{i}}^{t} \dot{x}(s) d s\right] } \\
\leq- & {\left[x(t)-x\left(t-h_{i}\right)\right]^{T} R\left[x(t)-x\left(t-h_{i}\right)\right] } \\
= & -x^{T}(t) R x(t)+2 x^{T}(t) R x\left(t-h_{i}\right) \\
& -x^{T}\left(t-h_{i}\right) R x\left(t-h_{i}\right)
\end{aligned}
$$

Therefore, we have

$$
\begin{aligned}
& E[\dot{V}(.)+2 \alpha V(.)] \\
& \leq E\left[x^{T}(t)\left[A^{T} P+P A+2 \alpha P-e^{-2 \alpha h_{1}} R-e^{-2 \alpha h_{2}} R+2 \rho_{1} I\right] x(t)\right. \\
& +2 x^{T}(t)\left[e^{-2 \alpha h_{1}} R\right] x\left(t-h_{1}\right)+2 x^{T}(t)\left[e^{-2 \alpha h_{2}} R\right] x\left(t-h_{2}\right) \\
& +x^{T}\left(t-h_{1}\right)\left[-e^{-2 \alpha h_{1}} R\right] x\left(t-h_{1}\right)+x^{T}\left(t-h_{2}\right)\left[-e^{-2 \alpha h_{2}} R\right] x\left(t-h_{2}\right) \\
& +x^{T}(t-h(t))\left[-S_{1} D+2 \rho_{2} I\right] x(t-h(t))+2 x^{T}(t-h(t))\left[S_{1}-S_{2} D\right] \dot{x}(t) \\
& \left.+\dot{x}^{T}(t)\left[S_{2}+S_{2}^{T}+h_{1}^{2} R+h_{2}^{2} R\right] \dot{x}(t)\right] \\
& =E\left[\zeta^{T}(t) \mathcal{M} \zeta(t)\right],
\end{aligned}
$$

where

$$
\zeta(t)=\left[x(t), x\left(t-h_{1}\right), x\left(t-h_{2}\right), x(t-h(t)), \dot{x}(t)\right] .
$$

Therefore, we finally obtain from (6) and the condition (4) that

$$
E[\dot{V}(.)+2 \alpha V(.)]<0, \quad \forall i=1,2, \ldots ., N, \quad t \in R^{+} .
$$


and hence

$$
E\left[\dot{V}\left(t, x_{t}\right)\right] \leq E\left[-2 \alpha V\left(t, x_{t}\right)\right], \quad \forall t \in R^{+} .
$$

Integrating both sides of (7) from 0 to $t$, we obtain

$$
E\left[V\left(t, x_{t}\right)\right] \leq E\left[V(\phi) e^{-2 \alpha t}\right], \quad \forall t \in R^{+} .
$$

Furthermore, taking condition (5) into account, we have

$$
E\left[\lambda_{1}\|x(t, \phi)\|^{2}\right] \leq E\left[V\left(x_{t}\right)\right] \leq E\left[V(\phi) e^{-2 \alpha t}\right] \leq E\left[\lambda_{2} e^{-2 \alpha t}\|\phi\|^{2}\right],
$$

then

$$
E[\|x(t, \phi)\|] \leq E\left[\sqrt{\frac{\lambda_{2}}{\lambda_{1}}} e^{-\alpha t}\|\phi\|\right], \quad t \in R^{+},
$$

which concludes the proof by Definition 1, concludes the proof of the theorem in the mean square.

Remark 2. Theorem 1 provides sufficient conditions for stochastic systems with interval time-varying delays (1) in terms of the solutions of LMIs, which guarantees the closed-loop system to be exponentially stable with a prescribed decay rate $\alpha$. The developed method using new inequalities for lower bounding cross terms eliminate the need for over bounding and provide larger values of the admissible delay bound. Note that the time-varying delays are non-differentiable, therefore, the methods proposed in [1-11, 17-25] are not applicable to system (1). The LMI condition (4) depends on parameters of the system under consideration as well as the delay bounds. The feasibility of the LMIs can be tested by the reliable and efficient Matlab LMI Control Toolbox $[27]$.

\section{Conclusion}

In this paper, we have proposed new delay-dependent conditions for the mean square exponential stability of stochastic systems with non-differentiable interval time-varying delay. Based on the improved Lyapunov-Krasovskii functionals and linear matrix inequality technique, the conditions for the mean square exponential stability of stochastic systems with interval time-varying delay have been established in terms of LMIs. 


\section{Acknowledgments}

This work was supported by the Thailand Research Fund Grant, the Commission for Higher Education and Faculty of Science, Maejo University, Thailand. The authors thank anonymous reviewers for valuable comments and suggestions, which allowed us to improve the paper.

\section{References}

[1] M. C. de Oliveira, J. C. Geromel, Liu Hsu, LMI characterization of structural and robust stability: the discrete-time case, Linear Algebra and its Applications, Vol. 296, 1999, 27-38.

[2] Kreangkri Ratchagit, STABILITY CRITERIA OF LPD SYSTEM WITH TIME-VARYING DELAY, International Journal of Pure and Applied Mathematics, Vol. 78, No. 6, 2012, 857-866.

[3] Kreangkri Ratchagit, STABILITY ANALYSIS OF LINEAR SYSTEMS WITH TIME DELAYS, International Journal of Pure and Applied Mathematics, Vol. 76, No. 1, 2012, 21-28.

[4] Kreangkri Ratchagit , STABILITY OF LINEAR TIME-VARYING SYSTEMS, International Journal of Pure and Applied Mathematics, Vol. 63, No. 4, 2010, 411-417.

[5] Kreangkri Ratchagit , EXPONENTIAL STABILITY OF SWITCHED LINEAR SYSTEMS, International Journal of Pure and Applied Mathematics, Vol. 58, No. 3, 2010, 361-371.

[6] K. Ratchagit , THE SUFFICIENT CONDITIONS FOR STABILITY OF LINEAR TIME-VARYING SYSTEMS WITH STATE DELAYS, International Journal of Pure and Applied Mathematics, Vol. 65, No. 1, 2010, $65-72$.

[7] K. Ratchagit and V.N. Phat, Stability criterion for discrete-time systems, Journal of Inequalities and Applications, 2010, 2010:201459 doi:10.1155/2010/201459.

[8] V.N. Phat, Y. Khongtham, and K. Ratchagit, LMI approach to exponential stability of linear systems with interval time-varying delays. Linear Algebra and its Applications, Vol. 436, 2012, 243-251. DOI: 10.1016/j.laa.2011.07.016 
[9] O.M. Kwon and J.H. Park, Delay-range-dependent stabilization of uncertain dynamic systems with interval time-varying delays, Applied Math. Conputation, Vol. 208, 2009, 58-68.

[10] H. Shao, New delay-dependent stability criteria for systems with interval delay, Automatica, Vol. 45, 2009, 744-749.

[11] Manlika Rajchakit, Piyapong Niamsup, Grienggrai Rajchakit, A constructive way to design a switching rule and switching regions to mean square exponential stability of switched stochastic systems with non-differentiable and interval time-varying delay, Journal of Inequalities and Applications, 2013, 2013:499. doi:10.1186/1029-242X-2013-499

[12] D. Yue and S. Won, Delay-dependent robust stability of stochastic systems with time delay and nonlinear uncertainties, Elect. Lett., Vol. 37, 2001, 992-993.

[13] Irada A. Dzhalladova, Jaromir Bastinec, Josef Diblik, and Denys Y. Khusainov, Estimates of Exponential Stability for Solutions of Stochastic Control Systems with Delay, Abstract and Applied Analysis, Article ID 920412, 14 pages, 2011.

[14] Manlika Rajchakit, Piyapong Niamsup, Grienggrai Rajchakit, A constructive way to design a switching rule and switching regions to mean square exponential stability of switched stochastic systems with non-differentiable and interval time-varying delay, JOURNAL OF INEQUALITIES AND APPLICATIONS, 2013. DOI: 10.1186/1029-242X-2013-499

[15] Grienggrai Rajchakit, Switching Design for the Asymptotic Stability and Stabilization of Nonlinear Uncertain Stochastic Discrete-time Systems, INTERNATIONAL JOURNAL OF NONLINEAR SCIENCES AND NUMERICAL SIMULATION, Vol. 14, 2013, 33-44. DOI: 10.1515/ijnsns2011-0176

[16] Hongli Dong, Zidong Wang, Daniel W. C. Ho, and Huijun Gao, , Robust $H_{\infty}$ filtering for Markovian jump systems with randomly occurring nonlinearities and sensor saturation: the finite-horizon case, IEEE Transactions on Signal Processing, Vol. 59, 2011, 3048-3057.

[17] G. Rajchakit, Robust stability and stabilization of nonlinear uncertain stochastic switched discrete-time systems with interval time-varying delays, APPLIED MATHEMATICS and INFORMATION SCIENCES, Vol. 6, 2012, 555-565. 
[18] Bo Shen, Zidong Wang, and Xiaohui Liu, Bounded $H_{\infty}$ Synchronization and State Estimation for Discrete Time-Varying Stochastic Complex Networks Over a Finite Horizon, IEEE Transactions on Neural Networks, Vol. $22,2011,145-157$.

[19] G. Rajchakit, Stabilization of switched discrete-time systems with convex polytopic uncertainties, Journal of Computational Analysis and Applications, Vol. 16, 2014, 20-29.

[20] Manlika Rajchakit, Piyapong Niamsup, Grienggrai Rajchakit, A constructive way to design a switching rule and switching regions to mean square exponential stability of switched stochastic systems with non-differentiable and interval time-varying delay, Journal of Inequalities and Applications 2013, 2013:499. doi:10.1186/1029-242X-2013-499

[21] Zidong Wang, Yao Wang, and Yurong Liu, Global Synchronization for Discrete-Time Stochastic Complex Networks With Randomly Occurred Nonlinearities and Mixed Time Delays, IEEE Transactions on Neural Networks, Vol. 21, 2010, 11-25.

[22] K. Ratchagit, V.N. Phat, Stability and stabilization of switched linear discrete-time systems with interval time-varying delay, Nonlinear Anal. Hybrid Syst., Vol. 5, 2011, 605-612. DOI: 10.1016/j.nahs.2011.05.006

[23] K. Gu, An integral inequality in the stability problem of time delay systems, in: IEEE Control Systems Society and Proceedings of IEEE Conference on Decision and Control, IEEE Publisher, New York, 2000.

[24] K. Ratchagit, Asymptotic stability of nonlinear delay-difference system via matrix inequalities and application, International Journal of Computational Methods, Vol. 6, 2009, 389-397. DOI: 10.1142/S0219876209001899

[25] P. Niamsup, M. Rajchakit, G. Rajchakit, Guaranteed cost control for switched recurrent neural networks with interval time-varying delay, JOURNAL OF INEQUALITIES AND APPLICATIONS, 2013. DOI: 10.1186/1029-242X-2013-292

[26] P. Niamsup, G. Rajchakit, New Results on Robust Stability and Stabilization of Linear Discrete-Time Stochastic Systems with Convex Polytopic Uncertainties, JOURNAL OF APPLIED MATHEMATICS, 2013. DOI: $10.1155 / 2013 / 368259$ 
[27] S. Boyd, L.El Ghaoui, E. Feron and V. Balakrishnan, Linear Matrix Inequalities in System and Control Theory, SIAM, Philadelphia, 1994. 
